\title{
Asteroids as calibration standards in the thermal infrared for space observatories ${ }^{\star, \star \star}$
}

\author{
T. G. Müller ${ }^{1}$ and J. S. V. Lagerros ${ }^{2}$ \\ 1 ISO Data Centre, Astrophysics Division, Space Science Department of ESA, Villafranca, PO Box 50727, \\ 28080 Madrid, Spain \\ 2 Astronomiska observatoriet, Box 515, 75237 Uppsala, Sweden \\ e-mail: Johan.Lagerros@astro.uu.se
}

Received 5 June 2001 / Accepted 19 October 2001

\begin{abstract}
Asteroids have been used extensively as calibration sources for the Infrared Space Observatory (ISO) and are planned to be used by future groundbased, airborne and space-based projects in the thermal infrared (IR) and in the sub-millimetre. We summarize the general IR observational parameters with a focus on space observatories and discuss brightness variations, apparent velocities and background influences. During the ISO mission ten wellstudied asteroids were used for the photometric calibration of ISOPHOT, but additionally the bright asteroids turned out to be of great interest for many technical tests and calibration aspects. We evaluated the different applications, like testing the photometry of the spectrometers, validation of relative spectral response functions, determination of beam profiles or colour correction tests. The description of the asteroids' thermal emission has been obtained by a recent thermophysical model (TPM). The important model aspects are size, albedo, shape together with the spin vector, a beaming model, thermal inertia and a wavelength-dependent emissivity. With a large sample of observational data provided by three different ISO instruments we had for the first time the possibility to study the thermal emission of several asteroids in detail. The intercomparison between results from different instruments allowed us to distinguish between observational errors and model shortcomings. It turned out that the accuracy of TPM predictions is in many cases strongly related to the limited knowledge of the asteroid shapes. The concepts of beaming, thermal inertia and wavelength dependent emissivities were nicely confirmed for a wide range of observing and illumination geometries under many aspect angles for different asteroids. The TPM predictions for Ceres, Pallas and Vesta are accurate within $5 \%$ over the full wavelength range from 5 to $200 \mu \mathrm{m}$, for Hygiea and a few other asteroids the predictions and observations agree within 10 to $15 \%$. We found similar emissivity behaviour for the four large asteroids over the full ISO wavelength range. Up to now, no clear spectral features have been seen in the asteroid far-IR spectra.
\end{abstract}

Key words. minor planets, asteroids - radiation mechanisms: thermal - infrared: solar system

\section{Introduction}

With the availability of the thermal IR wavelength range (from a few micron to the sub-millimetre range) through balloon, airborne and spaceborne instruments, it became necessary to establish new calibration standards and to develop new calibration strategies. In the early phases, instruments working in these wavelengths were usually calibrated against planets (mainly Mars, Uranus and

\footnotetext{
Send offprint requests to: T. G. Müller,

e-mail: Thomas.Mueller@esa.int

* Based on observations with ISO, an ESA project with instruments funded by ESA Member States (especially the PI countries: France, Germany, The Netherlands and the UK) and with the participation of ISAS and NASA.

$\star \star$ Tables A.1-A.3 and B.1-B.4 (Appendices A and B) are only available in electronic form at

http://www.edspsciences.org
}

Neptune), but with the development of more sensitive instruments these objects were too bright. Sandell (1994) made a first attempt to establish a set of secondary calibrators at sub-millimetre (submm) wavelengths. The emission from stellar photospheres is too faint in the far-IR and submm and therefore all calibrators were chosen from dust-rich sources, i.e. Ultra-Compact H II regions, protostars, protoplanetary nebulae and AGB-stars surrounded by massive dust envelopes. But often these sources are embedded in dust clouds which provide a strong and variable background (Sandell \& Aspin 1998). Additionally, modeling proves to be difficult and accurate far-IR extrapolations are almost impossible.

In the mid-IR, the connection from ground-based $N$ and $Q$ band photometry to the full mid-IR wavelength available from space was done via stellar models. In this way also the IRAS 12, 25 and $60 \mu \mathrm{m}$ bands were calibrated. 
But neither the stellar models nor planet model extrapolations were considered reliable for the $100 \mu \mathrm{m}$ band. Here, for the first time, asteroid models were used to "transport" the calibration from 60 to $100 \mu \mathrm{m}$ (Beichman et al. 1988).

The next big step in terms of sensitivity and thermal IR wavelength coverage was then achieved with the Infrared Space Observatory (ISO) (Kessler et al. 1996). ISO was equipped with four scientific instruments, covering the IR wavelengths from $2.5-240 \mu \mathrm{m}$. At a wavelength of $12 \mu \mathrm{m}$, ISO was one thousand times more sensitive and had one hundred times better angular resolution than its predecessor. During its routine operational phase (4 February 1996 to 8 April 1998) ISO successfully made over 26450 individual scientific observations ranging from objects in our own solar system right out to the most distant extragalactic sources, and approximately 4000 calibration observations. Especially in the far-IR beyond $50 \mu \mathrm{m}$ ISO was lacking reliable photometric standards. Uranus and Neptune at the bright end and the brightest stellar calibrators at the faint end left a gap of two orders of magnitude in flux. And, a special in-flight programme to establish secondary standards was not foreseen. Müller \& Lagerros (1998, in the following Paper I) provided a set of 10 asteroids, based on a recent thermophysical model code by Lagerros (1996a, 1997, 1998). The model input parameters were derived from ground-based mid-IR and submm observations and IRAS data. These sources have been extensively observed by ISO for photometric far-IR calibration, for testing relative spectral response functions and for many technical and calibration purposes.

In the following we summarize the important aspects of IR observational parameters and constraints for asteroids (Sect. 2). The crucial modeling issues are explained briefly (Sect. 3). In these two sections our goal is to provide useful information on the possibilities and limitations of using asteroids as calibration standards for space observatories. Section 4 gives an overview over the thermophysical model applications for ISO, including spectroscopic and photometric calibration aspects for different ISO instruments. It is meant as an illustration in what context and how asteroids can be utilized for other projects.

With the ISO data it was for the first time possible to test model predictions at far-IR wavelengths in a direct way. Photometrically useful observations of asteroids from ISOPHOT (Lemke et al. 1996), SWS (de Graauw et al. 1996) and LWS (Clegg et al. 1996) are presented and discussed. We summarize these new observations and the special data reduction procedures which were necessary to calibrate the observations exclusively against stars and planets (Sect. 5). The results clearly demonstrate the excellent quality of the models, but also the possible areas of improvement of the models for future missions with even higher requirements (Sect. 6). The new observational results from ISO photometry with ISOPHOT and LWS are listed in the Appendix.

\section{Asteroid IR observational aspects}

Asteroids are point-like $\left(<1^{\prime \prime}\right.$ angular diameter $)$ and emit the largest fraction of their energy at thermal IR wavelengths. The spectrum of an asteroid is dominated by reflected solar radiation in the visual and near IR, and by the thermal emission at longer wavelength. The transition region is narrow due to the steep slope in the Wien part of the thermal spectrum. Outside the transition region the contribution from either the reflected light or the thermal emission is completely negligible with respect to the other.

So far, no prominent features are known in the thermal spectra of asteroids at far-IR wavelength. At shorter wavelength, between 8 and $11 \mu \mathrm{m}$, broadband silicate features have been seen (Cohen et al. 1998; Dotto et al. 2000). Heras et al. (2000) report mid-IR emission signatures on Vesta, tentatively identified with olivine and pyroxene silicate groups. These features do influence groundbased $N$-band photometry but they are not relevant in the far-IR.

\subsection{Brightness variations}

The modeling of the thermal spectrum is reviewed in Paper I, but to a zeroth order the IR brightness is determined by the apparent size and the temperature across the surface. Over the orbital period (3-6 yr in the main-belt) the changing distance to the Sun will induce temperature variations covering the dynamic range approximately given by $\sqrt{(1+e) /(1-e)}$, where $e$ is the eccentricity of the orbit. From this the temperatures are typically $(17 \pm 13) \%$ higher in perihelion than in aphelion.

In principle the varying distance between the Earth and a main-belt asteroid (MBA), typically yields a factor of 5 between minimum and maximum flux density. In practice, however, there are constraints on the observing geometry, such as the solar elongation, which significantly reduces the dynamic range resulting from distance scaling. For ISO a factor of 2 was possible for MBAs in some extreme cases, but typical values were significantly smaller.

Asteroids rotate which produces a light curve, mainly due to the elongated shape and in some cases albedo variations across the surface. For the asteroids larger than $200 \mathrm{~km}$ in diameter, the range of periods is $4.2-29 \mathrm{~h}$ with a mean of $8.7 \mathrm{~h}$, and the mean amplitude of the visual light curve $0.22 \pm 0.15$ mag (Lagerkvist, private communication). The amplitude in the thermal IR is comparable to that in the visual, since both are approximately proportional to the projected surface area. Depending on the target and the application it may be important to consider the flux changes due to rotation, as given by the TPM, for integration times longer than about $0.5 \mathrm{~h}$.

\subsection{Apparent velocities}

Typical MBAs had between 0 and about $80 \mathrm{arcsec} /$ hour apparent speed, as seen from the Earth (vector addition of Earth and MBA movement). The ISO motion added in 


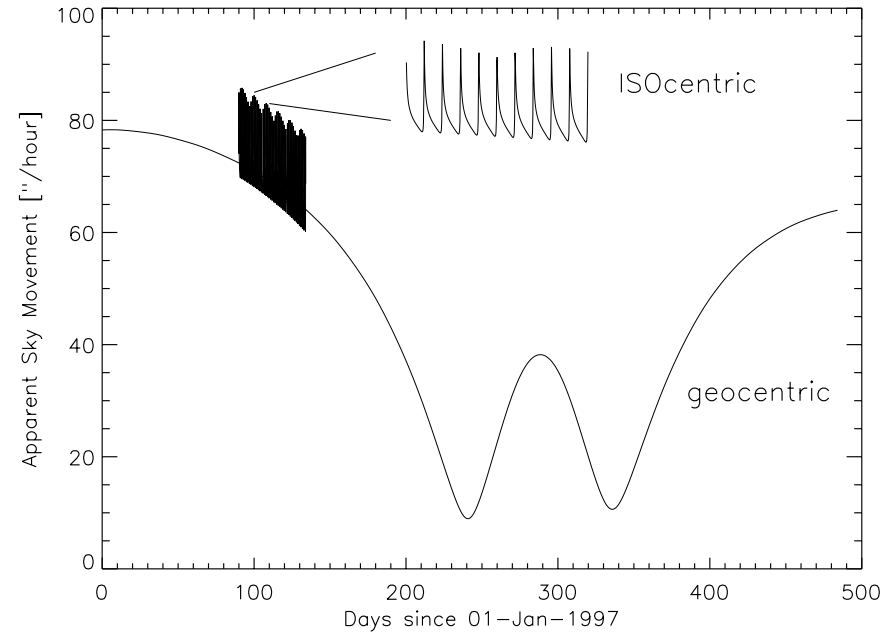

Fig. 1. The apparent sky movement ["/hour] of the asteroid Vesta with respect to the geocentre and ISO ( $24 \mathrm{~h}$ periodicity). ISO visibility periods are for $\lambda-\lambda \odot$ between 60 and $120^{\circ}$, corresponding to days 151 to 237 and 343 to 418 in the time scale of the graphics.

the worst case about 20 arcsec/hour at certain points of the $24 \mathrm{~h}$ orbit (see Fig. 1). Near Earth objects can have up to a few degrees/hour for close encounters with Earth.

Observations of solar system objects require therefore accurate, and in some cases, fast tracking. In case of ISO, tracking was done as a spacecraft raster with 2 arcsec step size, limited to apparent velocities of less then than $120 \mathrm{arcsec} / \mathrm{h}$ with respect to equatorial coordinates. This excluded fast moving objects like a few near Earth asteroids and comet Hyakutake from the ISO programme. The relative velocity between typical MBAs and Earth varies between 10 and $50 \mathrm{~km} \mathrm{~s}^{-1}$. The ISO orbit added an amplitude of about $10 \mathrm{~km} \mathrm{~s}^{-1}$ with a $24 \mathrm{~h}$ periodicity. The apparent sky motion of solar system objects has advantages and disadvantages, depending on the purpose of a measurement (see Sect. 4.4). In general, tracked satellite observations are usually more difficult than similar ones from the ground due to the involvement of gyros and reaction wheels.

\subsection{Infrared background}

The IR background in the ecliptic plane as seen from space is dominated by zodiacal light and therefore relatively smooth. It can be approximated as diluted black body radiation with temperatures ranging from $259 \mathrm{~K}$ (at $\left.\lambda-\lambda_{\odot}=120^{\circ}\right)$ to $280 \mathrm{~K}\left(\right.$ at $\left.\lambda-\lambda_{\odot}=60^{\circ}\right)$ (Abraham et al. 1999). Only at around $\lambda_{\text {ecl. }}=90^{\circ}$ and $\lambda_{\text {ecl. }}=270^{\circ}$, where the ecliptic crosses the galactic plane, the background strongly increases (see Fig. 2).

At $25 \mu \mathrm{m}$ the background along the ecliptic plane varies between 60 and $100 \mathrm{MJy} / \mathrm{sr}$ (as given by the COBE/DIRBE annual maps, corresponding to $\lambda-\lambda_{\odot}=$ $90^{\circ}$, Hauser et al. 1998) at $140 \mu \mathrm{m}$ the background values are around $10 \mathrm{MJy} / \mathrm{sr}$, but reaching up to $60 \mathrm{MJy} / \mathrm{sr}$ at $\lambda=90^{\circ}$ and more than $1500 \mathrm{MJy} / \mathrm{sr}$ at $\lambda=270^{\circ}$ (close to the galactic centre). In these places of high background the interstellar cirrus dominates at wavelengths longer than $100 \mu \mathrm{m}$.

The background of a given position in the ecliptic plane changes with time, depending on the angular distance from the Sun. Under the ISO visibility constraints, between $\lambda-\lambda_{\odot}=60^{\circ}$ and $\lambda-\lambda_{\odot}=120^{\circ}$, the background varied up to a factor of 3 at the mid-IR and about 10 to $30 \%$ at the far-IR. Preferable, low background configurations are therefore between $\lambda_{\text {ecl }}=0 \ldots 70^{\circ}, 100 \ldots 250^{\circ}$ and at $\lambda_{\text {ecl. }}=280 \ldots 360^{\circ}$ for $\lambda-\lambda_{\odot}>90^{\circ}$. Background values of 10,100 and $1000 \mathrm{MJy} / \mathrm{sr}$ correspond to approximately 2, 20 and $200 \mathrm{Jy}$ in a 100" circular aperture and to $0.02,0.2$ and $2 \mathrm{Jy}$ in a $10^{\prime \prime}$ aperture.

Although sky backgrounds can be high in some cases they only play a role for "cold" space observatories. Groundbased, airborne and passively cooled space instruments are limited by atmospheric and instrument selfemission. Sources are therefore measured in chopped mode and the sky background is negligible.

\section{Thermophysical modeling}

Using asteroids as calibrators requires a good understanding of the temporal flux variations on orbital and rotational time scales, as discussed above. Paper I and Müller et al. (1999) applied the thermophysical model developed by Lagerros (1996a, 1997, 1998), to a large data base of IR observations of a selection of previously well studied asteroids, in order to establish the asteroid standards used for ISOPHOT (Lemke et al. 1996). The model produces accurate thermal IR spectra and thermal lightcurves, taking into account a number of physical processes. Subjectively ordered by their relative importance, the main components of the model are:

- Observing and illumination geometry;

- Size and albedo;

- Emissivity as a function of wavelength;

- Surface roughness and beaming effect;

- Shape and spin state;

- Thermal inertia.

The fundamental parameter for far-IR model predictions is the object cross section at the time of the observation. Therefore, the size and shape and spin state uncertainties clearly dominate the model uncertainties. Tied to the size is the albedo because it determines the energy balance between reflected and absorbed solar radiation. In the model the emissivity was fitted to mid-IR and submm data and interpolated in the unexplored far-IR. Theoretically the beaming effect is much stronger in the mid-IR. For example, if the effect is constrained to a $10 \%$ level by data at $10 \mu \mathrm{m}$ it is typically within a $3-5 \%$-level at $50 \mu \mathrm{m}$, and $1-3 \%$-level at $200 \mu \mathrm{m}$. The thermal inertia causes the morning side to appear colder than the afternoon side, and some of the emission to occur on the night side (Morrison 1977; Hansen 1977; Lebofsky et al. 1986). 


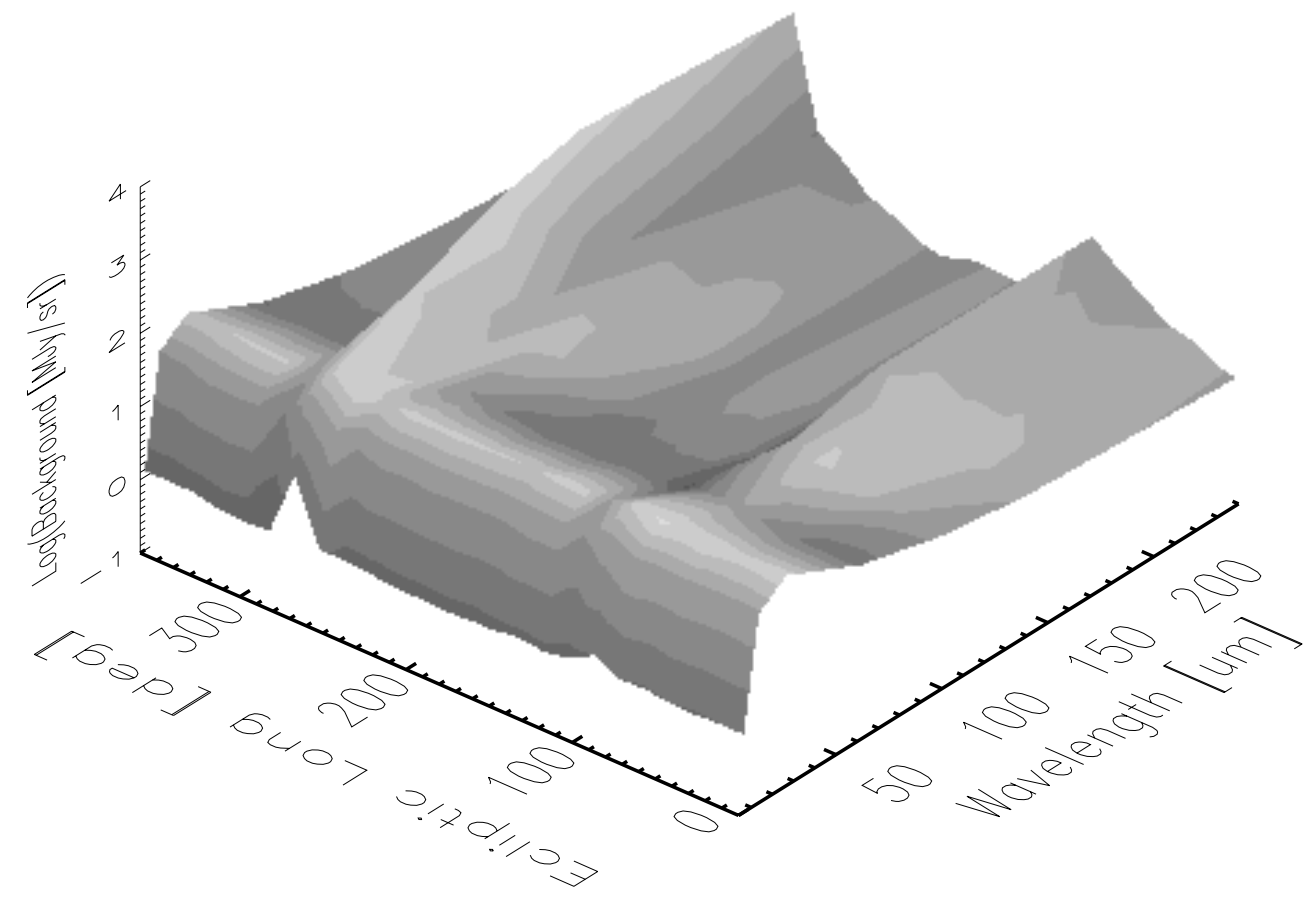

Fig. 2. The sky background in the ecliptic plane for a solar elongation of $\lambda_{\text {ecl. }}-\lambda_{\odot}=90^{\circ}$ at IR wavelengths. At $\lambda_{\text {ecl. }}=90^{\circ}$ and $\lambda_{\text {ecl. }}=270^{\circ}$ the ecliptic crosses the galactic plane. Note: the values are given in logarithmic scale.

Increasing the inertia decreases the amplitude of the thermal light curve. Varying the inertia introduces a significantly larger effect at shorter wavelengths, which are more sensitive to changes in surface temperature. Individual values of TPM input parameters are given in Paper I for 10 asteroids.

TPM spectral energy distributions and lightcurve predictions at any thermal wavelength can be requested from the authors via electronic mail. But reliable predictions are only possible for cases where the sizes and shapes are well known.

\section{TPM applications for ISO}

The asteroids were utilized in a wide range of calibration aspects ranging from absolute photometry, relative spectral response function checks to cross calibration and calibration of special modes. See also Müller \& Lagerros (2001) for a more technically oriented overview of all asteroid related calibration of ISO. The brief description of the main calibration aspects also illustrate the potential use of asteroids for future far-IR and submillimetre projects.

\subsection{Photometric calibrations of ISOPHOT in the far-IR}

A set of 10 asteroids was used during the ISO mission, together with bright standard stars and the planets Uranus and Neptune, to establish the photometric calibration of ISOPHOT at far-IR wavelength beyond $45 \mu \mathrm{m}$ (Schulz et al. 1999). Pallas was used also at shorter wavelength, down to $12 \mu \mathrm{m}$.
Figure 3 shows an example of the photometric calibration of ISOPHOT's internal reference source (FCS) against celestial standards for one pixel of the C200 camera in the $120 \mu \mathrm{m}$ filter. The signals of the FCS (expressed in terms of heating power) were ratioed to the signals produced by celestial standards, where the fluxes (or inband powers) were predicted from models. The model predictions for the asteroids were provided through Paper I. They established the calibration of the ISOPHOT FCS at intermediate flux level, whereas the stars served in the low range and the planets at the bright end of the detectors. In this way an absolute photometric calibration accuracy of $10 \%$ (P3), 15\% (C100) and 10\% (C200) was achieved for point sources close to the brightness level of the 10 asteroids (Klaas et al. 2000). Taking the general difficulties of opening a so far unexplored wavelength region beyond $100 \mu \mathrm{m}$ into account and considering the asteroid shape and thermal modeling aspects, the concept of using asteroids for the absolute calibration worked extremely well. Although the always changing background conditions for the asteroids required special attention and many dedicated measurements.

\subsection{LWS photometry and spectral response function}

Both the LWS absolute flux calibration and the relative spectral response function (RSRF) have been established using observations of Uranus in combination with a model (Griffin \& Orton 1993).

The photometry of LWS has been tested against Ceres, Pallas, Vesta and Hygiea repeatedly. The asteroids were considered as a viable possibility to ensure the 


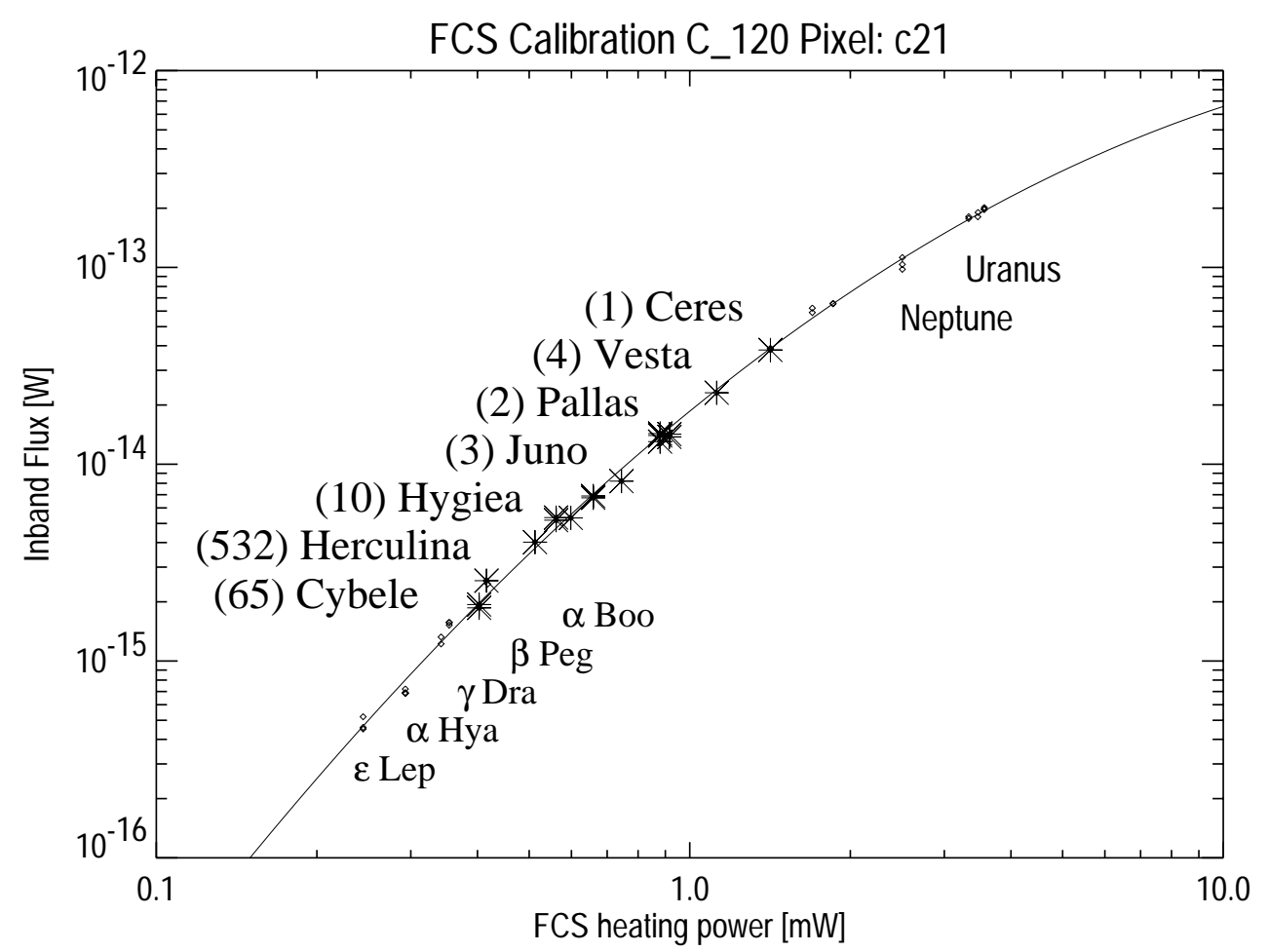

Fig. 3. The ISOPHOT calibration scheme. The stars cover the lowest flux range, the asteroids the intermediate one and the planets the high flux range. Individual measurements of celestial standards were used to calibrate the corresponding flux output of the internal calibration source (FCS), expressed in heating power.

calibration, since the brightest stellar standards are already too faint to obtain sufficient $S / N$ values. The bright asteroids provide approximately one tenth of the Uranus flux level (beyond $100 \mu \mathrm{m}$ ), whereas $\alpha$ Boo and $\alpha$ Tau are only at one hundredth of the Uranus brightness. The better flux coverage revealed non-linearities in the detector responses and allowed their empirical correction (Gry et al. 2001). Background contributions are not important for the bright planets, but had to be considered for the asteroids.

No prominent lines are known for the asteroids in the LWS wavelengths range. They therefore provide means of an independent RSRF determination. Photometric and spectroscopic asteroid measurements are currently analysed and the findings will be implemented in the LWS calibration scheme.

\subsection{SWS photometric and spectroscopic calibration}

Most of the SWS calibration programme were done on stars which perfectly cover the dynamic range of the detectors, except the highest brightness level beyond $12 \mu \mathrm{m}$. Here, bright asteroids and planets were used to check the final photometric and spectroscopic calibration (Morris 1999).

The grating flux calibration programme included Ceres, Pallas, Juno, Vesta, Hygiea together with the planets Saturn, Uranus and Neptune. For the Fabry-Perot interferometer calibration only measurements on Ceres and Pallas were used in addition to the stars.
Further qualitative comparisons related to spectral features or broadband shapes on the grating relative spectral response function included measurements on Ceres, Pallas, Juno and Hygiea. Also for the validation of different instrument configurations (SWS01/SWS06) a few asteroids were used (Leech et al. 2001).

\subsection{Miscellaneous applications and results}

Tracking and beam profiles: the apparent sky movements of three asteroids were used to validate the pointingtracking system of ISO. Additionally, the apparent sky movement of Vesta served in a special calibration programme to characterize the beam profile in dispersion direction on subarcsecond level. This complemented the extremely time consuming beam profile measurements (typically done with a series of satellite repointings on bright stars). In this way also the pointing uncertainties of the telescope could be minimized.

Colour correction and filter leaks: the accuracy of the photometric calibration depends strongly on the quality of the blocking elements and the characterization of the band passes. Ceres (in December 1997) and HR 6705 ( $\gamma$ Dra) had both approximately $150 \mathrm{Jy}$ flux density at $10 \mu \mathrm{m}$. In case of the asteroid, $10 \mu \mathrm{m}$ is still on the Wien-part of the spectrum with its energy peak at around $15 \mu \mathrm{m}$. For HR $670510 \mu \mathrm{m}$ lies on the Rayleigh-Jeans part of the spectrum with its peak wavelength in the visible. 
Table 1. Colour correction factors for blue $\left(T_{\mathrm{BB}}=5000 \mathrm{~K}\right)$ and red sources $\left(T_{\mathrm{BB}}=200 \mathrm{~K}\right)$ for 5 selected ISOPHOT filters.

\begin{tabular}{rccccc}
\hline & \multicolumn{5}{c}{ Detector-Filter } \\
$T_{\mathrm{BB}}$ & P1_3.6 & P1_12 & P2_25 & P3_60 & C100_120 \\
\hline $5000 \mathrm{~K}$ & 1.05 & 1.30 & 1.28 & 1.13 & 1.21 \\
$200 \mathrm{~K}$ & 1.69 & 0.89 & 1.05 & 1.07 & 1.17 \\
\hline
\end{tabular}

The measured in-band fluxes are therefore different and depend strongly on the width and shape of the band pass. These differences are clearly visible in the colour correction factors, which are calculated for a wide range of sources in comparison to a reference spectrum (IRAS and ISO used a constant energy spectrum $\nu F_{\nu}=$ const. as reference).

Table 1 gives an example of colour correction values between typical stellar and asteroid temperatures for different ISOPHOT filters. Indications for filter leaks are given when colour corrected monochromatic fluxes of one source type agree nicely with model predictions, but for the "opposite" source type they do not agree. ISOPHOT and also ISOCAM used asteroids for the blue versus red sources tests, to validate the colour correction factors and to investigate filter leaks.

ISOPHOT Serendipity Mode: Whenever possible, the longwavelength channel of the photometer was used during satellite slews. This ISOPHOT Serendipity Mode (e.g. Stickel et al. 1999) led to a partial sky survey, covering approximately $15 \%$ of the sky, at wavelengths of $170 \mu \mathrm{m}$, a spectral region not covered by the IRAS survey.

The bright asteroids, seen serendipitously during the ISO slews, allowed a flux calibration in a direct way, using TPM predictions (Müller et al., in preparation). This procedure improved the calibration significantly and extended the calibrated flux range to higher brightness levels, where many new galactic and extragalactic sources have been found (Stickel et al. 2000).

\section{New ISO observations of asteroids}

ISO used asteroids for calibration purposes in many contexts, but it also allowed an independent testing of TPM predictions in the unexplored far-IR. To avoid "selfcalibration" of the asteroid observations with a calibration based on asteroids, we developed special procedures to establish "asteroid free" calibration for the following data samples.

\subsection{Independent ISOPHOT observations}

With the method described in Paper I it was possible to exclude all asteroid observations from the ISOPHOT calibration scheme and to extract independently calibrated asteroid fluxes via the internal calibration source. Asteroid observations were in this way treated as normal
Table 2. Summary of LWS01 observations on asteroids.

\begin{tabular}{llll}
\hline TDT & JD (mid time) & Object & Scans \\
\hline 74803403 & 2450785.67361 & Ceres & 22 up \\
76903203 & 2450806.59514 & Ceres & 12 up \& 12 down \\
83201702 & 2450869.41667 & Hygiea & 10 up \& 10 down \\
\hline
\end{tabular}

scientific measurements, but now calibrated only against stars and planets. In total we derived 94 individual photometric data points between 50 and $200 \mu \mathrm{m}$ (Tables A.1-A.3). All values are background subtracted and colour corrected. The uncertainties are rms values of observational errors, calibration source model uncertainties and estimated method errors. Additionally, the TPM predictions are given in the tables. There are many more ISOPHOT observations of asteroids where our method could not be applied because of a lack of stellar and planetary calibrators in the specific instrument configuration. A detailed discussion per object follows in Sect. 6 .

\subsection{LWS AOT observations of asteroids}

\subsubsection{Full grating scans (LWS 01)}

The ISO Data Archive contains two full grating scans (observing mode LWS 01) on Ceres and one on Hygiea (see Table 2). Additional grating scans exist on 4 asteroids, but all taken in the photometrically unreliable LWS 99 mode.

The observations (OLP 10 products) have been analysed with ISAP $2.0^{1}$. An equal number of up and down scans for each detector were deglitched, sigma clipped $(2.5 \times \mathrm{rms})$ and averaged. Detectors SW1 to SW5 were then smoothed with the nominal resolution element of $0.29 \mu \mathrm{m}, \mathrm{LW} 1$ to LW5 with a resolution element of $0.6 \mu \mathrm{m}$ and both scan directions averaged. Observations on a large number of standard sources (planets, asteroids, stars) showed that the responsivities of the detectors LW1, LW2 and LW3 are flux dependent. Empirical correction values have been derived and were applied to the asteroid scans. The background values have been taken from COBEDIRBE (Hauser et al. 1998) weekly maps $(25-100 \mu \mathrm{m})$ and yearly maps $(140-240 \mu \mathrm{m})$, together with the LWS solid angles and the correction factors for extended sources. Maximal background contributions were $4 \%$ for Ceres and up to $50 \%$ for Hygiea with the largest influence at long wavelengths. One Ceres observation (74803403) was affected by straylight, which is noticeable in the noise of the long wavelength detector signals (Fig. 4).

The jumps are produced by the individual detector calibration. Additional artifacts of the RSRF can be seen at the edges of the detector bands. At longer wavelength, where the source signals are already comparable with the dark current, the noise level is much higher, but the overall

1 The ISO Spectral Analysis Package (ISAP) is a joint development by the LWS and SWS Instrument Teams and Data Centers. Contributing institutes are CESR, IAS, IPAC, MPE, RAL and SRON 


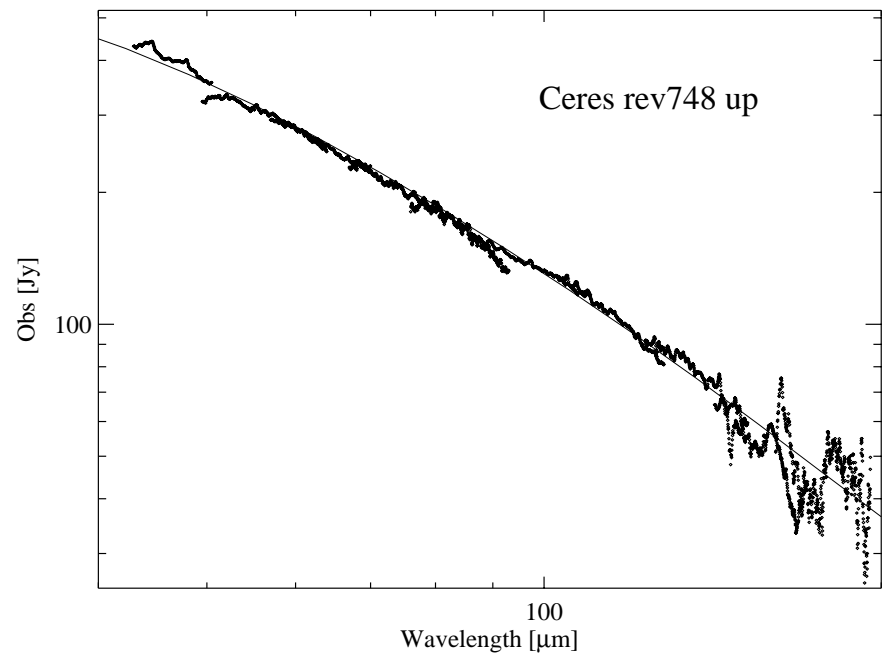

Fig. 4. Calibrated and background subtracted LWS spectrum of Ceres (3 Dec. 1997, revolution 748). Solid line: TPM prediction.

flux levels agree still quite well with the model predictions (the LW4 and LW5 are considered not to be photometrically reliable at this flux range). See also Sect. 6 for quantitative analysis of the observation to model ratios.

\subsubsection{Fixed grating positions (LWS 02)}

Ceres, Pallas, Vesta and Hygiea have been observed in the L02 narrow band mode, where 10 wavelengths were taken at the same time - one in each channel - with the grating remaining at a fixed position.

The data reduction was done manually by taking only the last $100 \mathrm{~s}$ of the measurements where the signals were usually stabilized. Dark current subtraction and flux calibration was done in the ISAP command mode. The detector non-linearities and the backgrounds have been corrected in the same way as for the LWS01 mode. Maximal background contributions were up to $16 \%$ for Ceres, up to about $10 \%$ for Pallas and Vesta and up to $40 \%$ for Hygiea. The dark current signals in LW4 $(160.6 \mu \mathrm{m})$ and LW5 $(178.0 \mu \mathrm{m})$ are in many cases unstable during the integration times and the corresponding fluxes could therefore not be used. Four Ceres and one Pallas L02 observations took place before ISO revolution 236 when the illuminator sequence for the instrument calibration was different. In these cases the calibration of the SW1 detector seems to be systematically off by 20 to $25 \%$ and fluxes are too low. No empirical correction has been done so far, but based on the asteroid results, this might be possible in the future. Tables B.1-B.4 summarize the observational results together with the model predictions. See also Sect. 6 for further discussions and the implications of these findings.

\subsection{SWS observations of 1 Ceres and 10 Hygiea}

Two sets of measurements (see Table 3) of Ceres and Hygiea have been analysed in a standard way, including

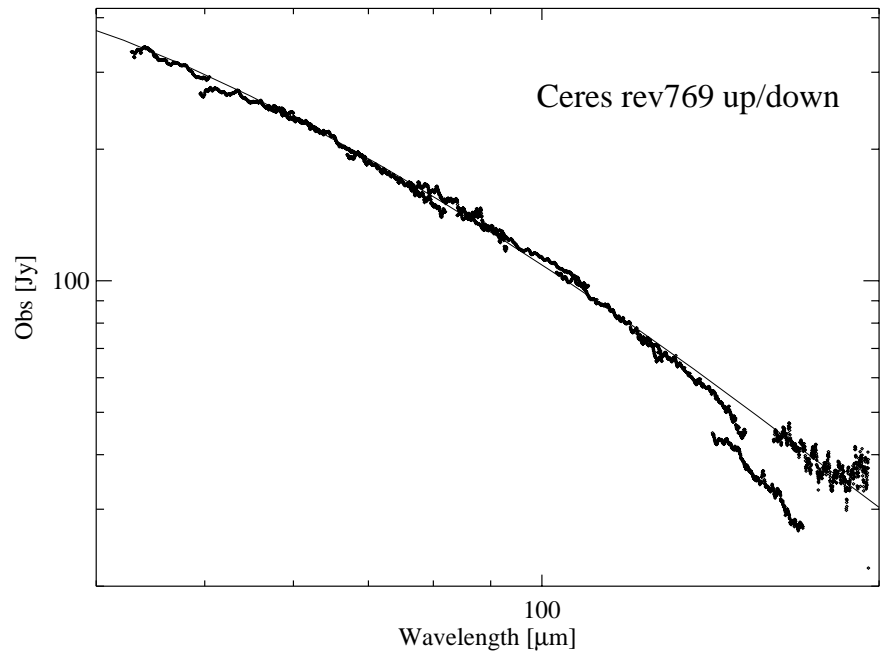

Fig. 5. Calibrated and background subtracted LWS spectrum of Ceres (24 Dec. 1997, revolution 769). Solid line: TPM prediction.

Table 3. Summary of 2 sets of SWS observations (SWS06) on Ceres and Hygiea. The nominal wavelength ranges of the SWS bands are [in $\mu \mathrm{m}$ ]: $1 \mathrm{~A}: 2.38-2.60 ; 1 \mathrm{~B}: 2.60-3.02$; $1 \mathrm{D}: 3.02-3.52$; 1E: $3.52-4.08 ; 2 \mathrm{~A}: 4.08-5.30 ; 2 \mathrm{~B}: 5.30-7.00 ; 2 \mathrm{C}: 7.0-12.0 ; 3 \mathrm{~A}$ : 12.0-16.5; 3C: 16.5-19.5; 3D: 19.5-27.5; 3E: 27.5-29.0; 4: 29.045.2 .

\begin{tabular}{llll}
\hline TDT & JD (mid time) & Object & Bands \\
\hline 71401706 & 2450751.78889 & Ceres & 2C, 3E, 4 \\
71401805 & 2450751.82361 & Ceres & 1D, 1E, 2A, 2B, 3C, 3D \\
71401904 & 2450751.85486 & Ceres & $1 \mathrm{~A}, 1 \mathrm{~B}, 3 \mathrm{~A}$ \\
67000903 & 2450707.67153 & Hygiea & $1 \mathrm{~B}, 3 \mathrm{~A}$ \\
67000904 & 2450707.72500 & Hygiea & $1 \mathrm{E}, 2 \mathrm{~A}, 2 \mathrm{~B}, 3 \mathrm{C}, 3 \mathrm{D}$ \\
67000905 & 2450707.77639 & Hygiea & $2 \mathrm{C}, 3 \mathrm{E}, 4$ \\
\hline
\end{tabular}

the latest tracking/beam profile corrections (OLP 10 products). No emphasis was put on the verification of spectral features, only the overall flux levels per band were of interest. Background values are usually below the 1-2\% level for the bright asteroids (see also Sect. 2.3) and were therefore neglected.

Figure 7 shows the observational results together with the model predictions on absolute scales. At short wavelengths the transition region between reflected light and thermal emission can be seen. A discussion on the quality of the TPM predictions per object is given in Sect. 6 .

\section{Discussion}

\subsection{Asteroids as technical calibrators for spacebased $I R$ projects}

The brightness level of the point-like asteroids, together with the high accuracy of the model predictions allow their usage as technical calibrators for space observatories in many aspects where high $S / N$ is needed. Even their 


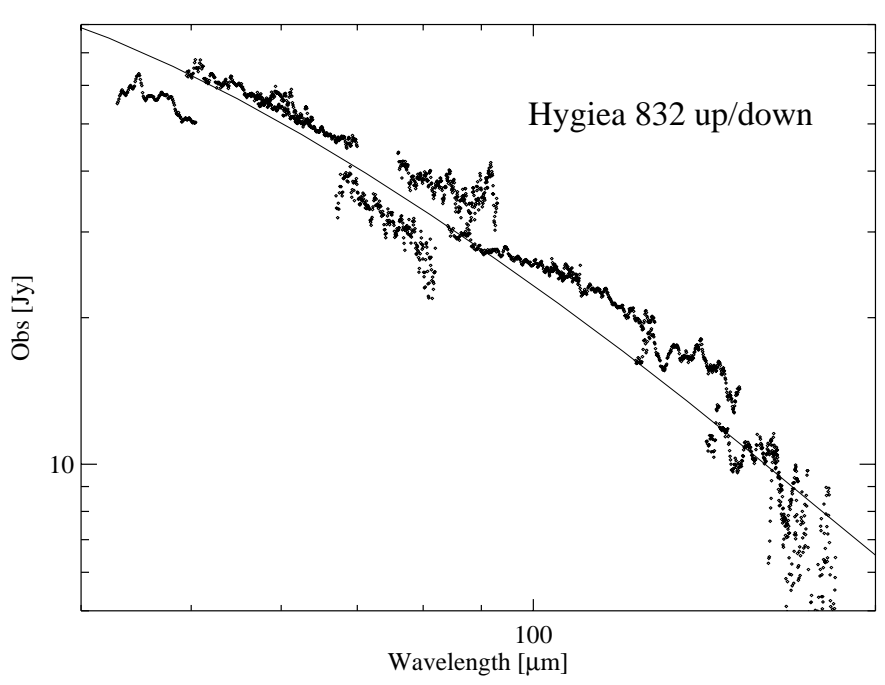

Fig. 6. Calibrated and background subtracted LWS spectrum of Hygiea (24 Feb. 1998, revolution 832). Solid line: TPM prediction.

apparent sky movement can be employed for the characterization of point-spread function, beam and aperture profile scans without moving the satellite.

Satellite tracking in combination with instrument apertures significantly smaller than the point-spread function can cause severe flux changes depending on the source position within the aperture. In some ISO configurations tracking was done with apertures smaller than $20^{\prime \prime}$ diameter which produced repeated flux changes for each tracking step.

The spectral energy distribution of main-belt asteroids with energy peaks at around $15 \mu \mathrm{m}$ makes them perfect counterparts to stellar calibrators, ideal to perform filter leak tests and to validate colour correction factors in the mid-IR.

The high ephemeris precision needed for modern stateof-the-art instruments is not an obstacle anymore. Recent $N$-body ephemeris software even allow satellite centric position calculations for solar system objects, including parallax corrections due to the satellite orbit. For the well known bright main-belt asteroids ephemeris accuracies of 1 arcsec are reached.

The always changing sky background conditions are clearly a disadvantage of asteroids. The high background areas, especially where the ecliptic and the galactic plane intersect, should be avoided. Depending on the size of the instrument aperture, far-IR space observations require investigations of the actual background conditions also outside the galactic plane.

All asteroids presented here are known to have only small amplitudes in their visual light curve (less than $0.23 \mathrm{mag}$ in the most extreme case, Lagerkvist et al. 1989). Depending on albedo, thermal parameters and observing geometry the thermal light curves have even smaller amplitudes. But long calibration observations of several hours might be affected by brightness changes of a few percent. But with the TPM it is possible to predict the light curve variations at any wavelength, based on shape and rotational aspects, and to take it into account for calibration observations.

The lack of prominent lines in the asteroid spectra make them also useful calibrators for the RSRF determination. Especially at far-IR and submillimetre wavelengths where the behaviour of stellar photospheres and planetary atmospheres is not perfectly well known, asteroids provide independent means to verify the spectroscopic calibration.

\subsection{The quality of the asteroid models}

\subsubsection{Observation to model ratios}

The independently derived ISO data allowed us to test the TPM quality. The observations have been brought together by calculating the corresponding TPM predictions and plotting the observation to model ratios.

The observation to model ratio representation (Figs. 8-13) include a large variety of observing geometries, aspect and phase angles, typically 2-3 orders of magnitude in flux and different background conditions for each asteroid. The observation from the 3 instruments (SWS, LWS, ISOPHOT) are taken in different observing modes (SWS06, LWS01, LWS02, PHT22, PHT25, PHT99) and different integration times, ranging from a few seconds (PHT) to hours (SWS). They underwent completely different data processing and calibration schemes. Nevertheless, the observation to model ratios give a consistent picture, expressed in ratios close to one. Deviations from the model are caused by different effects. In case of the ISOPHOT, the uncertainties in the background determination and in the calibration dominate the final errors. For SWS and LWS the background contributions are of minor importance. Instead problems at the band edges appear. The SWS band 4 in addition has a wrong slope and LWS detectors LW4 and LW5 are affected by dark current problems. Some deviations are due to model uncertainties, mainly by shape and size approximations. Systematical offsets in Figs. 8-13 indicate incorrect sizes (for example Juno), standard deviations (Tables 4, 5) larger than typical observational errors indicate problems with the shape approximation by ellipsoids (for example Hygiea). Within the error bars the observations agree extremely well with the model predictions over all wavelengths and at all flux levels. Clearly visible features (up to $5 \%$ over the continuum) exist only at around $10 \mu \mathrm{m}$ (see also Dotto et al. 2000). More detailed spectroscopic analysis in the future might reveal additional structures at levels below $5 \%$.

\subsubsection{Results per instrument}

Before the quality of individual asteroid models are discussed, we analysed the results per instrument to see if different calibration concepts lead to the same results.

ISOPHOT: the statistical analysis of the ratios between observations and model predictions, are given in Table 4. 

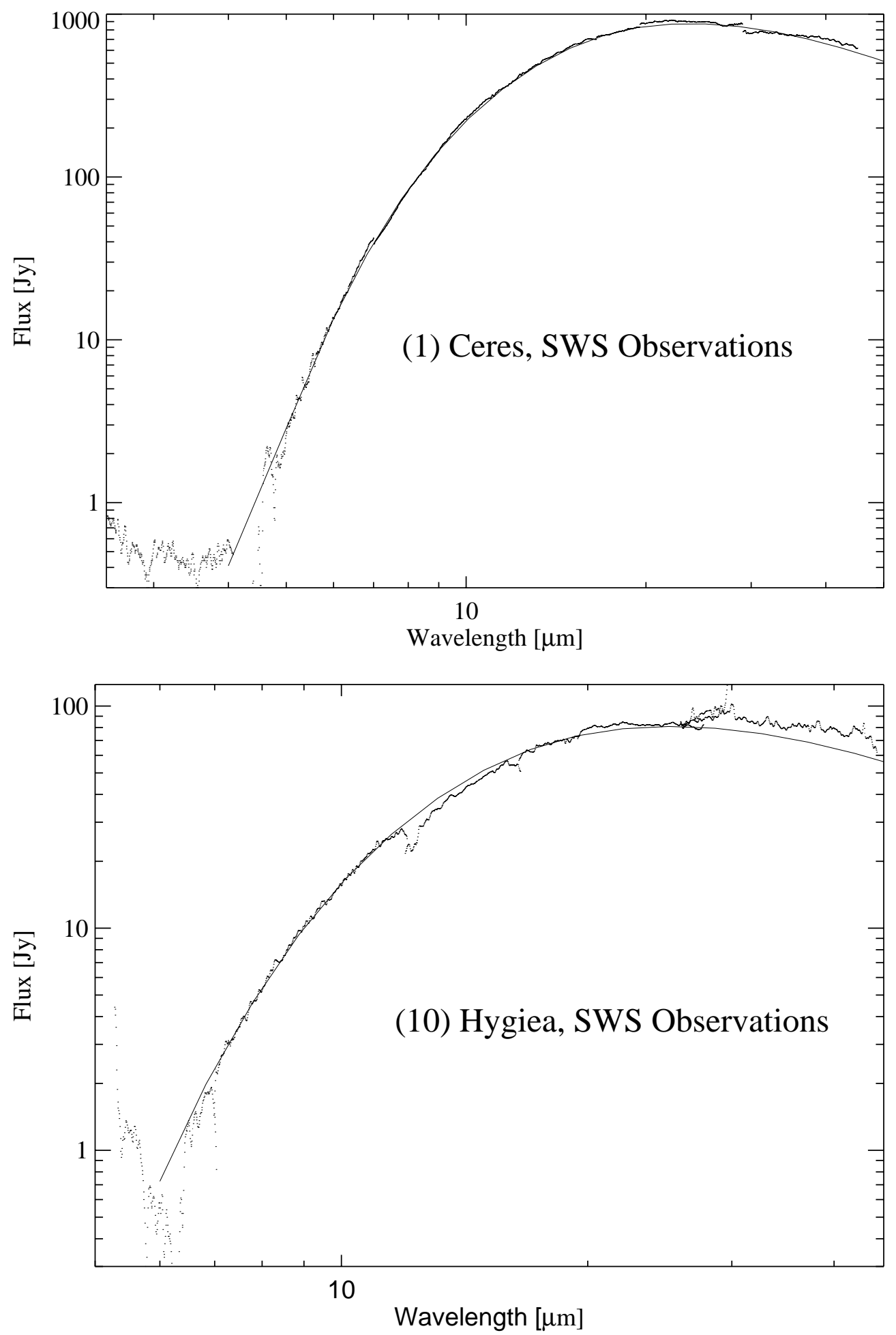

Fig. 7. SWS observations of Ceres and Hygiea. The TPM predictions are plotted as solid lines.

In general observations and model predictions agree on absolute level very well over the whole ISOPHOT wavelength range. In case of Juno the model predictions seem to underestimate and in the case of Herculina to overestimate the observed fluxes. The difficulty to calibrate these measurements with stars and planets only (see Sect. 5) is reflected in the large scatter of the ratios. Additionally, the uncertainties in the shape model might contribute to the scatter for some objects.
LWS: the ratios between LWS 01 observations of Ceres and the corresponding TPM predictions per detector range between 0.94 and 1.04 , with a weighted mean of $0.99 \pm 0.03$. For Hygiea the individual ratios vary between 0.83 and 1.22 , with a weighted mean of $1.04 \pm 0.15$ (Figs. 4-6, 9 and 13). Here, as in case of the SWS measurements, the band average are the relevant numbers for the comparison. The band slope and band edge effects are 


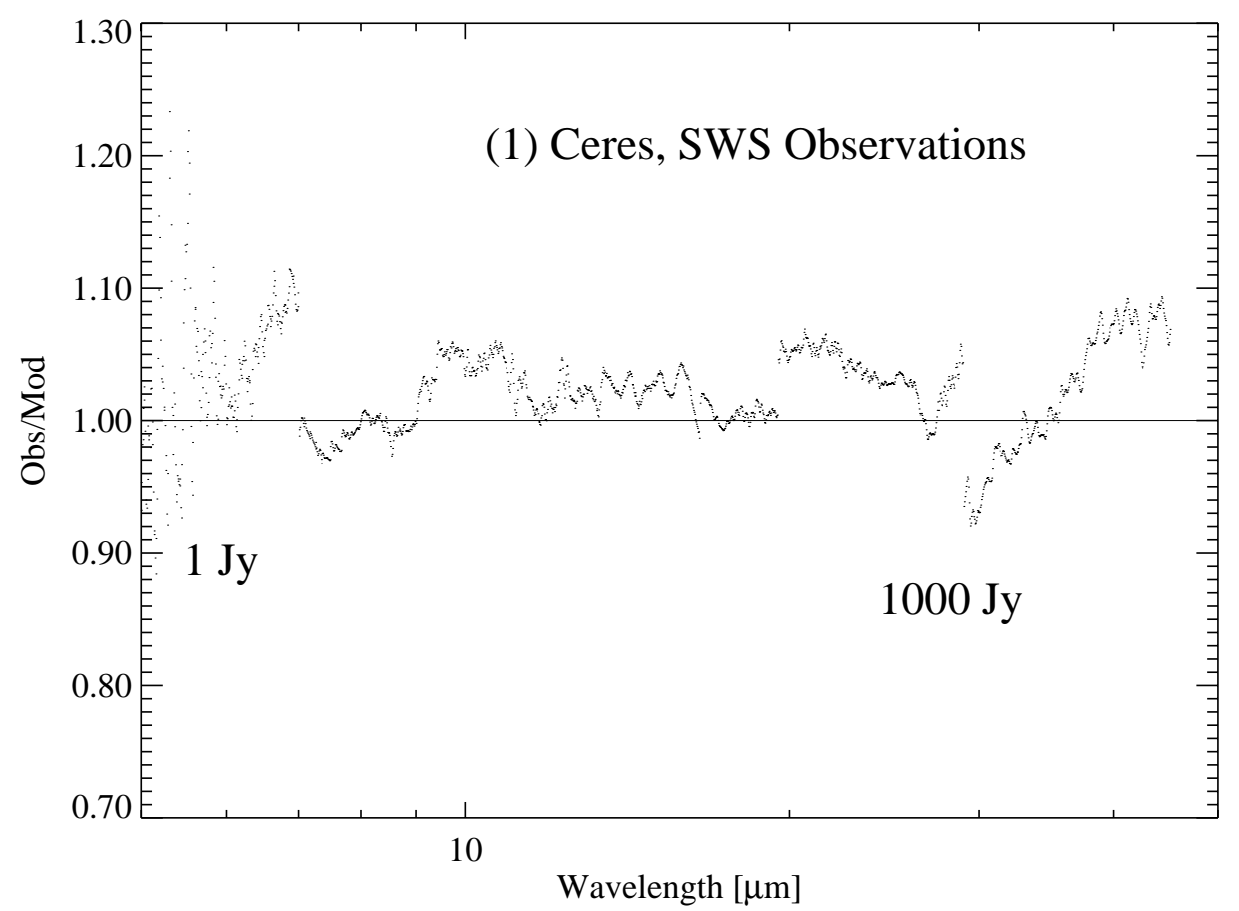

Fig. 8. Ceres SWS observation divided by the TPM prediction. Jumps between SWS bands and the slope of band 4 are calibration artifacts. At around $10 \mu \mathrm{m}$ broad band Silicate features are visible.

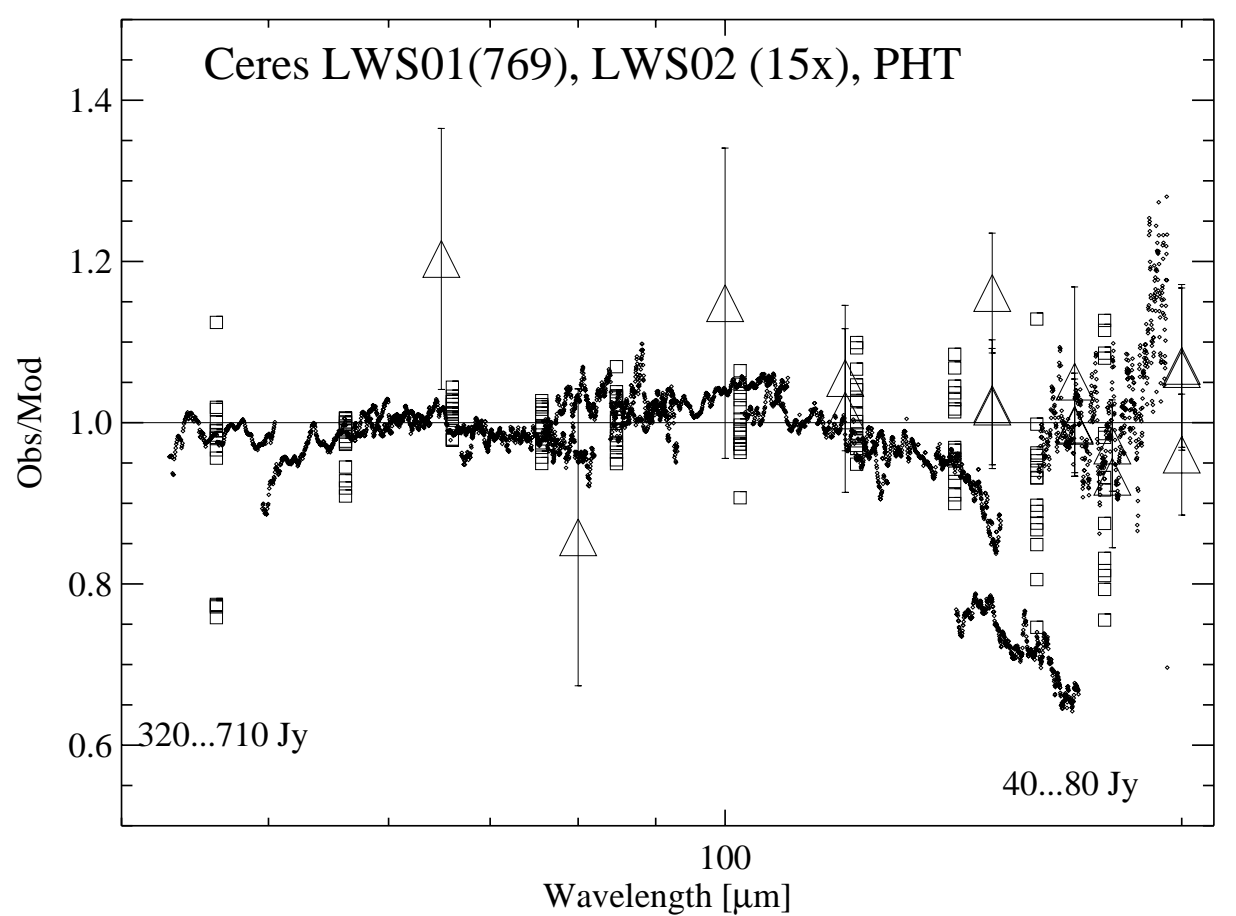

Fig. 9. Ceres LWS (fixed grating: squares; grating scans: dots) and ISOPHOT (triangles) observations divided by the corresponding TPM predictions. The LWS LW4 detector scan is off by about $25 \%$ for unknown reasons. No systematic differences between LWS and ISOPHOT can be seen.

of minor interest for the model tests. The photometry of the LW4 and LW5 detectors was not reliable due to dark current problems.

The general agreement between the photometric observations (L02) and models (Table 5) is better than $5 \%$ for Ceres, Pallas and Vesta and better than 15\% for Hygiea.
The two fixed grating measurements (squares in Fig. 13) follow the same jumps as the individually scanned bands (dots). We believe that this is due to dark current effects at the low flux level for Hygiea.

SWS: the ratios per detector between SWS observations of Ceres and TPM predictions range between 1.01 


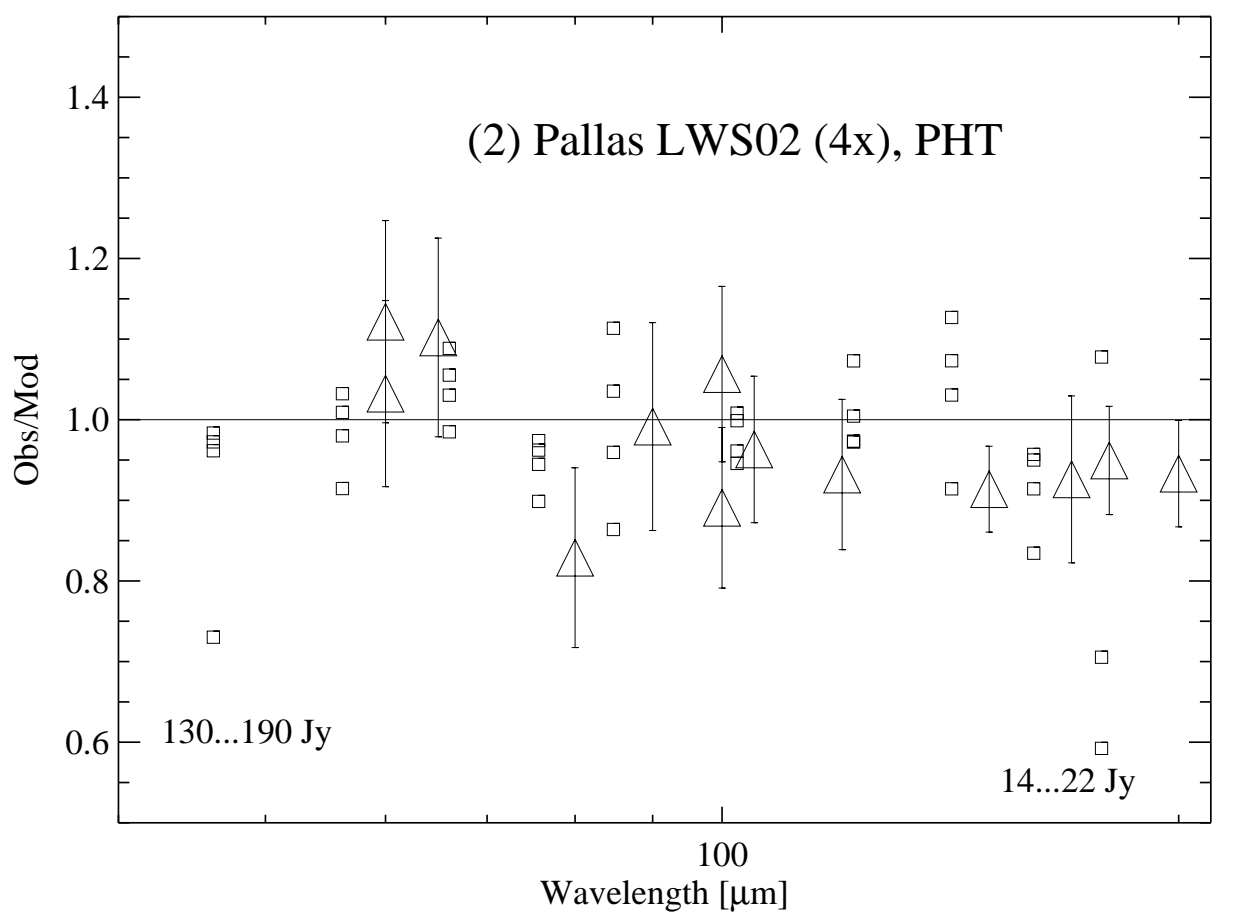

Fig. 10. Pallas LWS (fixed grating: squares) and ISOPHOT (triangles) observations divided by the corresponding TPM predictions. No systematic differences between LWS and ISOPHOT can be seen.

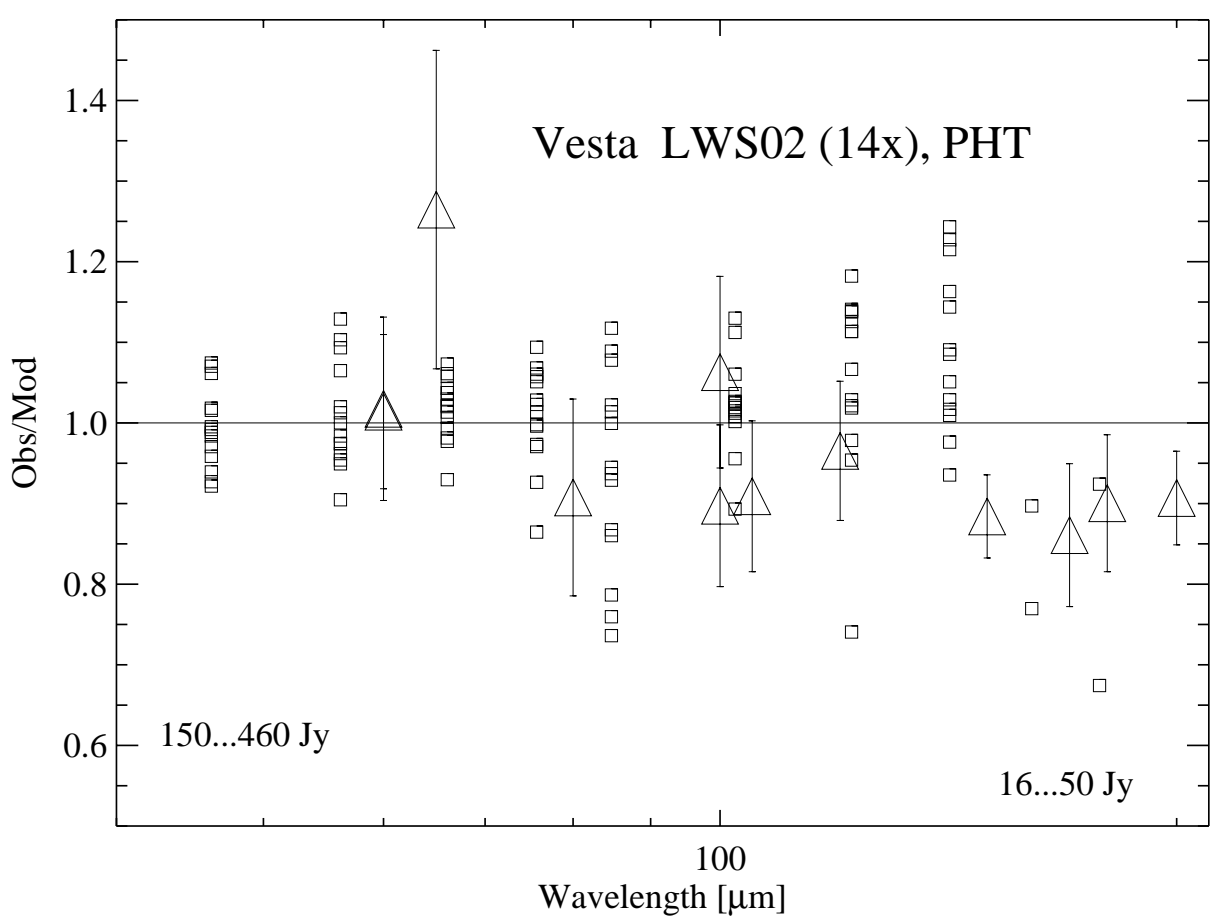

Fig. 11. Vesta LWS (fixed grating: squares) and ISOPHOT (triangles) observations divided by the corresponding TPM predictions. No systematic differences between LWS and ISOPHOT can be seen.

and 1.05, with a weighted mean of $1.02 \pm 0.01$. For Hygiea the ratios vary between 0.85 and 1.18 , with an weighted mean of $1.03 \pm 0.10$ (Figs. 7, 8 and 12). SWS band 4 is photometrically difficult due to the deviating band slope, which seems to occur for all solar system objects. The Hygiea band $2 \mathrm{~b}$ is affected by the low level flux.
No systematic offsets on absolute terms between the instruments or observing modes can be seen. The jumps between SWS and LWS detector bands are instrumental artifacts which are not yet perfectly understood, but photometrically reliable are in general only the values at the key wavelengths of the bands. 


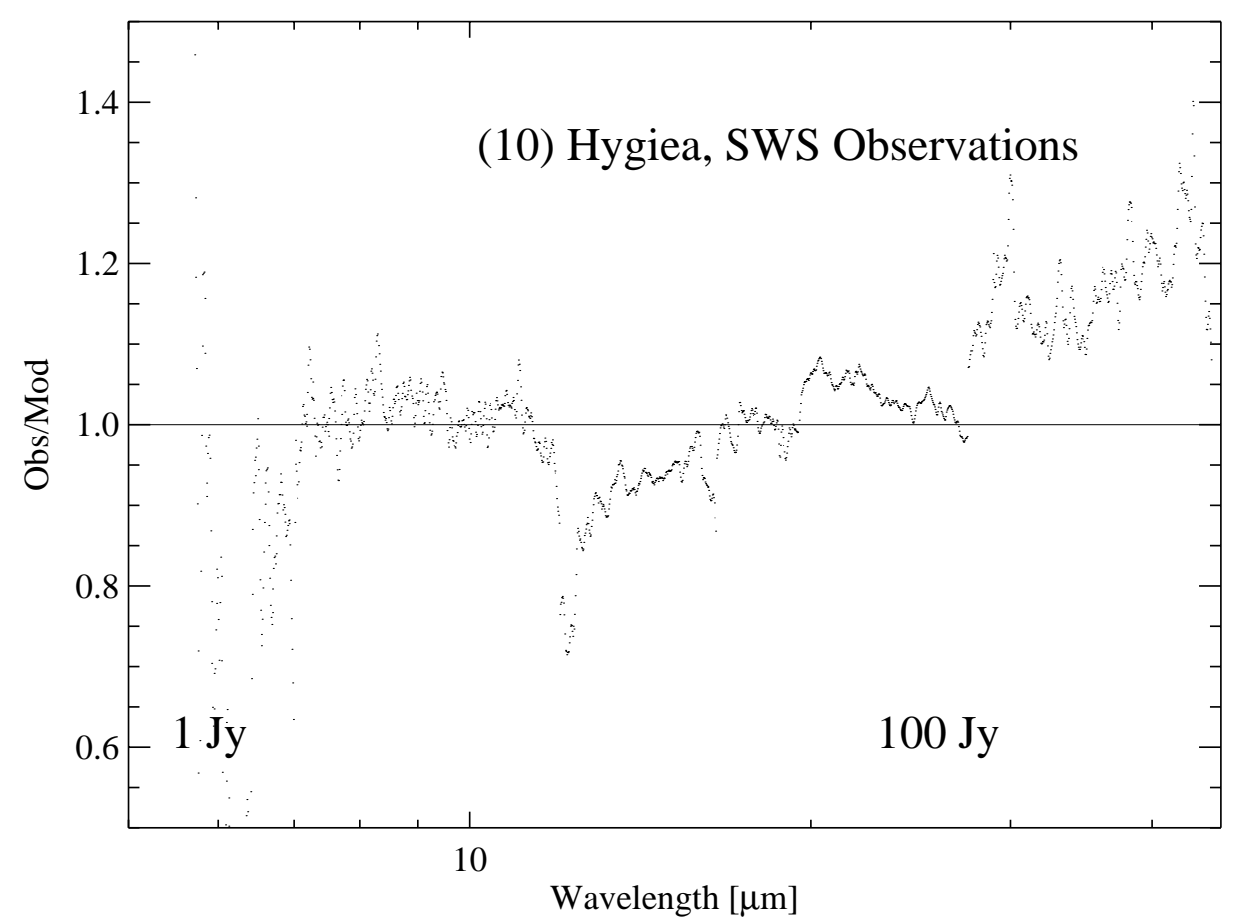

Fig. 12. Hygiea SWS observation divided by the TPM prediction. Jumps between SWS bands and the slope of band 4 are calibration artifacts.

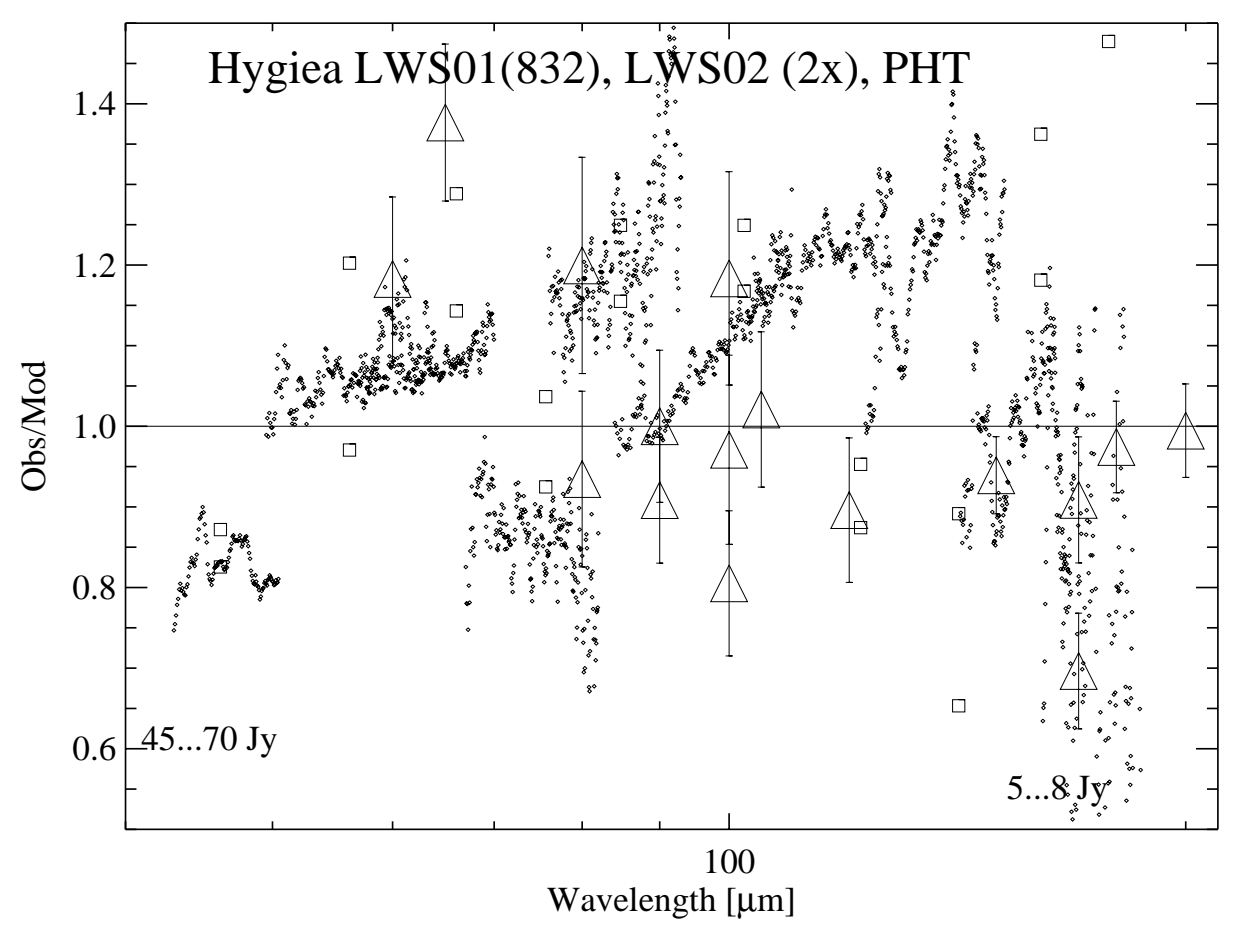

Fig. 13. Hygiea LWS (fixed grating: squares; grating scans: dots) and ISOPHOT (triangles) observations divided by the corresponding TPM predictions. Jumps between LWS bands are calibration artifacts. Especially the high quality ISOPHOT C200 measurements are consistent with the TPM predictions.

\subsubsection{Model quality per asteroid}

Not all individual asteroid models have the same quality. Already in Paper I the 10 asteroids were divided in primary and secondary calibrators, depending on the quality and coverage of the available observational data and on the reliability of the size/shape values. A similar separation can also be seen in Figs. 8-13 with observations from ISOPHOT, SWS and LWS. 
Table 4. Averaged ratios between ISOPHOT observations and model predictions. The total number of observations varies between 3 and 16 per object. All measurements are taken between $60 \mu \mathrm{m}$ and $200 \mu \mathrm{m}$.

\begin{tabular}{lrll}
\hline Asteroid & No of Meas. & Mean Ratio & Std.Dev. \\
\hline 1 Ceres & 16 & 1.03 & 0.08 \\
2 Pallas & 13 & 0.97 & 0.08 \\
3 Juno & 11 & 1.13 & 0.10 \\
4 Vesta & 12 & 0.97 & 0.11 \\
10 Hygiea & 16 & 1.00 & 0.16 \\
54 Alexandra & 3 & 0.98 & 0.11 \\
65 Cybele & 13 & 1.04 & 0.10 \\
532 Herculina & 10 & 0.93 & 0.07 \\
\hline
\end{tabular}

Table 5. Ratios between LWS 02 observations and TPM predictions, weighted mean values and standard deviations per detector (SW1, 2, 3, 4, 5 and LW1, 2, 3) and per asteroid. The photometry of the LW4 and LW5 detectors was not reliable due to dark current problems.

\begin{tabular}{|c|c|c|c|c|}
\hline $\begin{array}{r}\lambda \\
{[\mu \mathrm{m}]}\end{array}$ & $\begin{array}{c}\text { Ceres } \\
15 \text { Obs. } \\
\text { Obs/Mod }\end{array}$ & $\begin{array}{c}\text { Pallas } \\
4 \text { Obs. } \\
\text { Obs/Mod }\end{array}$ & $\begin{array}{c}\text { Vesta } \\
14 \text { Obs. } \\
\text { Obs/Mod }\end{array}$ & $\begin{array}{c}\text { Hygiea } \\
2 \text { Obs. } \\
\text { Obs/Mod }\end{array}$ \\
\hline 46.2 & 1 & .12 & 0.99 & 0.86 \\
\hline 56.2 & $0.97 \pm$ & 55 & 1.01 & 1.09 \\
\hline 66.1 & $1.01 \pm 0.02$ & $1.04 \pm 0.04$ & $1.02=$ & 1.23 \\
\hline 75.7 & $0.99 \pm$ & $0.94 \pm 0.03$ & $1.01=$ & 0.98 \\
\hline 84.8 & $1.00=$ & $0.99=$ & 0.94 & 1.22 \\
\hline 102.4 & $1.00=$ & $0.98=$ & $1.02=$ & 1.08 \\
\hline 122.2 & $1.01 \pm 0.05$ & $1.01 \pm 0.05$ & $1.05 \pm 0.11$ & $1.10 \pm 0.07$ \\
\hline 141.8 & $0.99 \pm 0.06$ & $1.04 \pm 0.09$ & $1.04 \pm 0.22$ & $1.05 \pm 0.23$ \\
\hline Mean & $.00=$ & $.98=$ & $1.01=$ & 0.98 \\
\hline
\end{tabular}

1 Ceres: based on a large set of ISO observations from 3 different instruments the model predictions agree within $3 \%$ at wavelength between 5 and $200 \mu \mathrm{m}$ (Tables 4 , 5 and Figs. 8, 9). The larger scatter in the ISOPHOT data is related to the calibration procedure. Submm and millimetre data proved that the Ceres TPM works also out to about $2 \mathrm{~mm}$ (Paper I). Ceres is therefore the best modeled asteroid and an excellent far-IR calibration standard. The TPM concept (Lagerros 1996a, 1997, 1998) and the model input parameters (Müller et al. 1999) are confirmed. Caution is only necessary for $N$-band photometry (see SWS band 2C in Fig. 8) where the spectral behaviour from 8 to $11 \mu \mathrm{m}$ suggests the presence of silicates on the surface (Dotto et al. 2000). Detailed spectroscopic analysis might reveal other low level spectral features at longer wavelengths.

2 Pallas: the 13 ISOPHOT and the 4 LWS fixed grating observations are within $3 \%$ of the predictions. Individual measurements with large deviations can be tracked down in most cases to difficulties in the calibration (see for example LWS SW1 problem mentioned in Sect. 5).
The shape values of Pallas are uncertain and our parameters deviate from the occultation and speckle results (Paper I). The observation to model ratios close to one seem to confirm our parameters, but some scatter might be due to deviations from the real physical shape of Pallas. For this asteroid a 3-dimensional shape from time delay and Doppler frequency radar measurements (Ostro 1993) would be of great importance.

4 Vesta: the 12 ISOPHOT and the 14 LWS grating observations are within $3 \%$ of the predictions. The scatter in Fig. 11 is large and a few individual observations are off by $25 \%$. This might be an indication that the physical shape model could be improved, although the highest quality 3 dimensional HST shape (Stooke 1997) has been used. The albedo variations on the surface (Binzel et al. 1997) have not been taken into account (Müller \& Lagerros 1998) and might cause deviations in one or the other direction, depending on the rotational phase. The ISOPHOT data points beyond $150 \mu \mathrm{m}$ are systematically below 1.0. This indicates that the emissivity drop to 0.6 in the submm (Redman et al. 1998; Redman et al. 1992) might start already in the far-IR.

10 Hygiea: all data sets agree on average within a few percent with the model predictions. Nevertheless, the model predictions are only accurate to about $15 \%$ for a given time. The reason lies mainly in the uncertainties of the shape model. Based on lightcurve observations the axis ratio of the ellipsoidal shape in Paper I was assumed to be $a / b=1.29$ and $b / c=1.18$. Erikson (2000) stated now that a meaningful determination of $b / c$ was not possible. Based on IRAS data the effective diameter would be $407.1 \mathrm{~km}$ (Tedesco et al. 1992), we found in Paper I $429.9 \mathrm{~km}$. Our shape model produced at the time of the SWS observation an apparent effective diameter of $448 \mathrm{~km}$ and in consequence a too high model flux. Forcing the apparent effective diameter to our previous value of $429.9 \mathrm{~km}$ gave much better agreement with the SWS observations (Fig. 7), except for band 4 where the calibration is problematic (Barucci et al. 2001). Here again, a radar based shape model could improve the situation significantly.

Other asteroids: for the asteroids Juno, Alexandra, Cybele and Herculina we have only ISOPHOT far-IR observations to test the model quality. Additionally, their diameters are based on radiometric methods only (Paper I). Radar observations and direct imaging (HST, Speckle) would improve the size and shape models and put the models on more solid ground.

For Juno the eleven ISOPHOT measurements show that the model systematically underestimated the derived fluxes by more than $10 \%$. Since the direct diameter measurements and the radiometric diameters agree almost perfectly (Paper I), the source of the deviations is not known. Independent fluxes from the submm would 
allow to test our model values, especially the emissivity behaviour and the radiometric diameter. Erikson (2000) published an ellipsoidal shape model which differs from the one we have used. Additional lightcurve, thermal and/or radar observations could also reveal the cause of the deviations.

The 3 ISOPHOT observations of Alexandra agree nicely, but a direct size measurement and an improved shape model would lower the model uncertainties.

The Cybele model agrees on average within $4 \%$ with all 13 ISOPHOT measurements, but has a $10 \%$ uncertainty for individual measurements. The radiometric size (Paper I) and the shape and spin vector solution (Erikson 2000) are not very reliable and could be improved by additional measurements. Using the $9 \%$ lower IRAS diameter in the TPM would produce an average ratio of 0.85 for the 13 ISO observations. Here again, an improved shape from other observing techniques would lower the uncertainties.

Herculina's shape was derived from lightcurve observations (Michalowski 1996). The combination of occultation measurements (Bowell et al. 1978), speckle observations (Drummond et al. 1985) and radiometric analysis (Paper I) agreed resonably well. Nevertheless, the model predictions are systematically too high. Our previous classification as primary calibrator cannot be kept anymore and individual model predictions can be off by $15 \%$ in some extreme cases.

In summary, we classify now only Ceres, Pallas and Vesta as primary calibrators with an estimated model uncertainty of $5 \%$, although individual predictions can be off by as much as $10 \%$. All other object models have larger uncertainties. We estimate the TPM prediction accuracy to about 10 to $15 \%$ with the possibility that individual calculations might be off by as much as $20 \%$. The reliable TPM wavelengths range can be given with 5 to $200 \mu \mathrm{m}$ for well known main-belt asteroids. At the submm and millimetre range the TPM can be used, but with lower accuracy (Paper I).

\subsection{Model input parameters}

Size/shape: the sizes and shapes of the asteroids determine the absolute flux level. Since the observation to model ratios are all close to 1.0 we conclude that the specified asteroid sizes are close to the true values. Nevertheless, some of the diameters need confirmation from direct size methods, like imaging, speckle, occulation or radar techniques. The object shapes, and therefore also the cross sections at a given time, are more complex. Approximating the shape as a 3-dimensional ellipsoid reproduces the visible and thermal light curves to a large extent, but some deviating model predictions might be due to the simplification of the shapes in combination with uncertain ellipsoid ratios of the semi-major axis.

Albedo variations: albedo changes on the asteroid surface of $\pm 10 \%$ between different hemispheres would only produce far-IR flux changes of about $\pm 1 \%$. Thus, even from high quality taken over a full rotation period it would be difficult to determine these variations. Rotationally resolved mid-IR observations would be better suited. Although the influence of albedo variations in the far-IR is of minor importance, in case of Vesta it would improve the model quality.

Emissivity: in Paper I the wavelength dependent emissivity was derived from mid-IR and submm observations. All asteroids show a similar behaviour in the mid-IR, with emissivities between 0.9 and 1.0. At longer wavelength a clear trend of decreasing emissivity can be seen, reaching values of 0.83 at $200 \mu \mathrm{m}$ and 0.8 in the submm. In case of Vesta, submm emissivities as low as 0.6 are found (Redman et al. 1992, 1998). But due to a lack of observations it was not clear where and in what way the emissivity changes in the far-IR. To have better possibilities to investigate the emissivity behaviour in the unknown far-IR range we used exactly the same emissivity model for all asteroids. And in fact it is difficult to distinguish Vesta from the others by looking at the ratio figures in the ISO far-IR wavelength range. There is a trend in Figs. 10 and 11 with lower emissivities for Pallas and Vesta beyond $130 \mu \mathrm{m}$, but more observations are needed to clarify that aspect. We conclude therefore that Vesta's emissivity has to drop within a short wavelength range closer to the submm from 0.8 to 0.6 , possibly related to grain size effects and/or Vesta's composition. Laboratory measurements of different possible asteroid surface materials are currently underway (Brucato, priv. communications). This might be an additional possibility to investigate the emissivity behaviour in the thermal-IR.

Light curve variations: the thermal light curve variations are predicted by the TPM based on shape models and the thermal behaviour. But only limited observational material is available to test the model predictions. The here presented ISO observations contain also 13 consecutive LWS L02 measurements over a full rotation period (5.34 hours) of Vesta. The TPM predicts a light curve amplitude of $3.8 \%$ for this epoch at the LWS wavelengths range. The normed mean values of all 10 LWS detectors varied at the same time between $1.01 \pm 0.03$. But distinguishing LWS observational uncertainties from effects due to the asteroid rotation is difficult at this low level. The same is true for the analysis of the light curve phase: the large scatter between the results from individual detectors does not give a consistent result. A combination with ISOPHOT observations taken simultaneously might give a clearer picture (Schulz et al. in preparation).

\subsection{Outlook}

The ISO project and recent requests from far-IR and submm projects clearly show the need for new calibration sources. The large asteroids can close this gap under the 
above specified limitations of variability, background influences and the individual TPM uncertainties. There are still many open questions and aspects which have to be addressed in the future. The asteroid shape models together with the absolute size and albedo values are crucial for the TPM and can be improved. First indications of small features in the asteroid spectra have already been seen. But what materials do they correspond to? Would it be possible to include them in a radiative transfer code to be able to explain the empirical emissivity values? Work on identifying the spectral features in the mid-IR is on going and first empirical results have been published (Dotto et al. 2000; Barucci et al. 2001). In the far-IR beyond $30 \mu \mathrm{m}$ the extraction of spectral features below a $5 \%$ level is more difficult and hardly any laboratory data exist for comparison. For the fainter asteroids in the ISO programme there are more unknowns and larger uncertainties in the TPM input parameters. Instead of connecting them only to stars and planets, one could use in addition the bright asteroids to recalibrate observation and to improve our knowledge about them. Establishing new sources as a reference with better known model parameters would also be an alternative and a possibility to enlarge our small sample of new far-IR standards. The radar techniques are especially promising to improve the quality of existing standards and to establish new ones.

Acknowledgements. We thank Cecile Gry, Martin Burgdorf, Bernhard Schulz (ISO Data Centre in Villafranca), Fred Lahuis (Dutch ISO Data Analysis Centre) and Tanja Lim (Rutherford Appleton Laboratory) for the helpful discussions and support in LWS, SWS and ISOPHOT data reduction. We also would like to express our thanks to the referee, Dr. G. Sandell. His comments were very helpful and improved the paper significantly.

\section{Appendix A: Independent ISOPHOT Observations}

\section{A.1. ISOPHOT P3 Observations}

\section{A.2. ISOPHOT C100 Observations}

\section{A.3. ISOPHOT C200 Observations}

\section{Appendix B: Independent LWS Observations}

\section{B.1. LWS02 Observations on 1 Ceres}

\section{B.2. LWSO2 Observations on 2 Pallas}

\section{B.3. LWSO2 Observations on 4 Vesta}

\section{B.4. LWS02 Observations on 10 Hygiea}

\section{References}

Abraham, P., Leinert, C., Acosta-Pulido, J. A., Schmidtobreick, L., \& Lemke, D. 1999, in The Universe as Seen by ISO, ESA SP-427, ed. P. Cox, \& M. F. Kessler, 261
Barucci, A., Dotto, E., Brucato, J. R., et al. 2001, Icarus, submitted

Beichman, C. A., Neugebauer, G., Habing, H. J., Clegg, P. E., \& Chester, T. J. 1988, Infrared astronomical satellite (IRAS) catalogs and atlases, vol. 1: Explanatory supplement

Binzel, R. P., Gaffey, M. J., Thomas, P. C., et al. 1997, Icarus, 128, 95

Bowell, E., McMahon, J., Horne, K., et al. 1978, BAAS, 10, 594

Clegg, P. E., Ade, P. A. R., Armand, C., et al. 1996, A\&A, 315, L38

Cohen, M., Witteborn, F. C., Roush, T., et al. 1998, AJ, 115, 1671

Dotto, E., Müller, T. G., Barucci, A., et al. 2000, A\&A, 358, 1133

Drummond, J. D., Hege, E. K., Cocke, W. J., et al. 1985, Icarus, 61, 232

Erikson, A. 2000, Ph.D. Thesis Freie Universität Berlin, DLRForschungsbericht 2000-37, ISSN, 1434-8454

de Graauw, Th., Haser, L. N., Beintema, D. A., et al. 1996, A\&A, 315, L49

Griffin, M., \& Orton, G. S. 1993, Icarus, 105, 537

Gry, C., Swinyard, B., Harwood, A., et al. 2001, The ISO Handbook: LWS - The Long Wavelength Spectrometer, ESA SAI/99-077/Dc, Version 1.2

Hansen, O. L. 1977, Icarus, 31, 456

Hauser, M. G., Kelsall, T., Leisawitz, D., \& Weiland, J. (Eds.) 1998, COBE Diffuse Infrared Background Experiment (DIRBE) Explanatory Supplement, Version 2.3, COBE Ref. Pub., No. 98

Heras, A., Morris, P., Vandenbussche, B., et al. 2000, ASP Conf. Ser., 196, 205

Kessler, M. F., Steinz, J. A., Anderegg, M. E., et al. 1996, A\&A, 315, L27

Klaas, U., Laureijs, R. J., Radovich, M., Schulz, B., \& Wilke, K. 2000, ISOPHOT Calibration Accuracies, ESA SAI/1998-092/Dc

Lagerkvist, C.-I., Harris, A. W., \& Zappala, V. 1989, in Asteroids II, ed. Binzel, Gehrels, \& Shapley Matthews (Arizona University Press), 1162

Lagerros, J. S. V. 1996a, A\&A, 310, 1011

Lagerros, J. S. V. 1997, A\&A, 325, 1226

Lagerros, J. S. V. 1998, A\&A, 332, 1123

Lebofsky, L. A., Sykes, M. V., Tedesco, E. F., et al. 1986, Icarus, 68, 239

Lebofsky, L. A., \& Spencer, J. R. 1989, in Asteroids II, ed. Binzel, Gehrels, \& Shapley Matthews (Arizona University Press), 128

Leech, K., de Graauw, T., van den Ancker, M., et al. 2001, The ISO Handbook: SWS - The Short Wavelength Spectrometer, ESA SAI/2000-008/Dc, Version 1.1

Lemke, D., Klaas, U., Abolins, J., et al. 1996, A\&A, 315, L64

Magnusson, P., Barucci, A., Drummond, J. D., et al. 1989, in Asteroids II, ed. Binzel, Gehrels, \& Shapley Matthews (Arizona University Press), 66

Michalowski, T. 1996, A\&A, 309, 970

Morris, P. 1999, SWS Photometric Calibrations at EOM, ESA, ISO internal report, http://www.iso.vilspa.esa.es

Morrison, D. 1977, Icarus, 31, 185

Müller, T. G., \& Lagerros, J. S. V. 1998, A\&A, 338, 340 (Paper I) 
Müller, T. G., Lagerros, J. S. V., Burgdorf, M., et al. 1999, ESA SP-427, in The Universe as Seen by ISO, ed. P. Cox, \& M. F. Kessler, 141

Müller, T. G., Dotto, E., \& Barucci, A. 2000, ESA SP-456, in ISO Beyond the Peaks, ed. A. Salama, M. F. Kessler, K. Leech, \& B. Schulz, 33

Müller, T. G., \& Lagerros, J. S. V. 2001, ESA SP-481, in The Calibration Legacy of the ISO Mission, in press

Ostro, S. J. 1993, Planetary radar astronomy, Reviews of Modern Physics, 65, 1235

Redman, R. O., Feldman, P. A., Matthews, H. E., Halliday, I., \& Creutzberg, F. 1992, AJ, 104, 405

Redman, R. O., Feldman, P. A., \& Matthews, H. E. 1998, AJ, 116, 1478

Sandell, G. 1994, MNRAS, 271, 75
Sandell, G., \& Aspin, C. 1998, A\&A, 333, 1016

Schulz, B., Huth, S., Kinkel, U., et al. 1999, ESA SP-427, in The Universe as Seen by ISO, ed. P. Cox, \& M. F. Kessler, 89

Stickel, M., Bogun, S., Lemke, D., et al. 1998, A\&A, 336, 116 Stickel, M., Lemke, D., Toth, L. V., et al. 1999, in ESA SP427 , in The Universe as Seen by ISO, ed. P. Cox, \& M. F. Kessler, 839

Stickel, M., Lemke, D., Klaas, U., et al. 2000, A\&A, 359, 865

Stooke, P. J. 1997, http://europa.geog. uwo.ca/stooke_personalhomepage

Tedesco, E. F., Veeder, G. J., Fowler, J. W., et al. 1992, The IRAS Minor Planet Survey, Phillips Laboratory 